\title{
RHEOLOGY OF SOLID METHANE AND NITROGEN: APPLICATIONS TO TRITON
}

\author{
Janusz Eluszkiewicz and David J. Stevenson \\ Division of Geological and Planetary Sciences \\ Californis Institute of Technology
}

\begin{abstract}
The existing information about the theological poperties of solid methane and nitrogen is reviewed. The rain limitation is the absence of creep data for solid nitrogen, bet estimated properties are derived on the basis of simple scling arguments for van der Waals bonded materials. On Trion solid methane and most likely nitrogen are too soft to and any topography over billions of years. Topographic mport by water ice-rich materials seems almost certain. Nitogen and methane do not intermix by solid-state diffusion. The weakmess (absence?) of the opposition effect on Triton can be explained by annealing of nitrogen grains, provided the berve sizes characteristic of freshly deposited frost.
\end{abstract}

\section{Introduction}

The Voyager encounter with Triton has revealed a bewildering variety of geological structures [Smith et al. 1999]. The UV experiment onboard the spacecraft combined with Earth-based spectroscopical observations provide evidence that solid methane and nitrogen are the dominant. coastituents at least on the southern polar surface currently faing the Sun, although the very low partial pressure of $\mathrm{CH}_{4}$ (anbsurution by about a factor of 10, [Broadfoot et al 1989]) in the atmosphere implies that the fractional coverage of Sunfacing regions covered with $\mathrm{CH}_{4}$ must be low. An monstanding of the theological properties of these ices at low emperatures (the surface temperature on Triton is $~ 37 \mathrm{~K}$ ) therefore to be a prerequisite in any attempt to explain Triton's morphology and history. The aim of this note is to marize the existing data on theology of $\mathrm{CH}_{4}$ and $\mathrm{N}_{2}$ ices and provide a preliminary assessment of the implications of data For methane this will lead to the construction of a deformation map. When used in homologous stress and emperature variables, this map should also have some aplicability for nitrogen, for which no creep data exist. Albough there exists no definite proof of this, it seems phusible that materials of similar crystal structure and molecular bonding should have similar deformation maps in wese coordinates. Both nitrogen and methane are van der Wals-bonded solids. Based on the theological information so drained we will then proceed to evaluate its importance in explaining three phenomena related to Triton: 1. Intermixing wethane and nitrogen and its implications for volatile radistribution; 2. The preservation of ropography; and 3. The encess of the opposition effect.

Coprright 1990 by the American Geophysical Dnion.

Toper mimer 90GL01472

0014-8276/90/90GL-01472\$03.00

\section{Construction of the deformation maps}

In modeling the flow phenomena on planetary surfaces the first step is to identify the mechanisms involved in deformation. The total strain-rate is then the sum of contributions from individual mechanisms. The main deformation mechanisms are [Ashby and Verall 1978]:

1. Cataclastic flow caused by repeated cleavage fracture and rolling of already fractured fragments. In this paper this process will be characterized by a single parameter, the fracture strength $\sigma_{f}$.

2. Low temperature plasticity. Although not usually considered a steady-state mechanism, this process is included here to determine the onset of time-dependent deformation, with the corresponding yield stress $\sigma_{\mathbf{y}}$.

3. Diffusional flow, including volume (Nabarro-Herring) and boundary (Coble) diffusion. The corresponding flow is Newtonian viscous. The associated strain-rate is $\varepsilon_{3}=$ $42 D_{\text {eff }} \sigma \Omega / \mathrm{kTd}^{3}$, where $D_{\text {eff }}=D_{v}\left[1+(\pi \delta / d)\left(D_{b} / D_{v}\right)\right], D_{v}=$ $D_{\text {ov }} \exp \left(-E_{w} / R T\right)$, and $D_{b}=D_{o b} \exp \left(-E_{b} / R T\right)$ are the effective, volume, and boundary diffusivities, respectively, $E_{v}$ and $E_{b}$ are the corresponding activation energies, $\sigma$ is the deviatoric stress, $T$ temperature, $\Omega$ the molecular volume, $k$ the Boltzmann constant, $R$ the universal gas constant, $d$ the grain size, and $\delta$ the thickness of the boundary diffusion path.

4. Power-law creep, with strain-rate commonly expressed as $\dot{\varepsilon}_{4}=A \sigma^{n} \exp \left(-Q_{d} / R T\right)$.

5. Other processes, e.g., pressure solution creep and creep aided by dynamic recrystallization. These processes are less well understood and moreover their importance for Triton is difficult to assess. They will therefore be excluded from the present considerations, which leads to an underestimate of the total strain-rate. However, it is shown below that deformation by power-law creep alone is in most cases geologically rapid so this omission is unlikely to cause severe problems.

Except for cataclastic flow, deformation is largely independent of pressure at the modest pressures of interest. This leads to a converient way of displaying rheological properties of a material in the form of deformation maps. A deformation map shows regions in the $\sigma, T$-plane in which a particular deformation mechanism dominates. The boundaries between regions are found by equating the strain-rates for each pair of mechanisms and solving the resulting equations for the function $\sigma(T)$. On those boundaries are superimposed contours of constant strain-rate, given by equations of the form $\mathrm{e}=\mathrm{const}=\mathrm{f}(\sigma, \mathrm{T})$. The homologous coondinetes are o/ and $T / T_{m}$, where $\mu$ is the shear modulus and $T_{m}$ is the melting temperature.

\section{Methane}

Rheological data for methane are more complese then for 
nitrogen. The fracture strength has been measured by Haynes [1971] at $T=77 \mathrm{~K}$ with the result $\sigma_{\mathrm{f}}=7.46$ bar. In the deformation maps the line $\sigma=\sigma_{\mathrm{f}}$ is taken as the boundary above which flow takes place by repeated fracture. The possible dependence of $\sigma_{f}$ on $T$ is neglected but this is unlikely to be a serious limitation, especially an a log-log plot. The yield stress of methane was determined in the $40-50 \mathrm{~K}$ range relevant to Triton by Leonteva et al. [1970], $\sigma_{\mathrm{y}} \sim 5$ bar. The parameters for volume diffusion, obtained from an analysis of the measurements of the spin-lattice relaxation time $T_{1}$ in the NMR experiemnts, are $D_{o v}=10^{-3} \mathrm{~m}^{2} / \mathrm{s}$ and $E_{v}=15.9 \mathrm{~kJ} / \mathrm{mole}$ [Chezeau and Strange 1979]. The molecular volume is $\Omega=$ $5.11 \times 10^{-29} \mathrm{~m}^{3}$. No data for boundary diffusion exist and therefore following the suggestion by Ashby and Verall [1978] the scaling $E_{b}=2 / 3 E_{v}$ and $\delta D_{o b}=10^{-9} D_{o v}$ (in SI units) will be used. The creep parameters at temperatures relevant to Triton have been measured by Bolshutkin et al. [1968]: $n=3$, $\mathrm{Q}_{\mathrm{c}}=8.4 \mathrm{~kJ} / \mathrm{mole}$, and $\mathrm{A}=10 \mathrm{MPa}^{-3} \mathrm{~s}^{-1}$. Two words of caution are in order concerning these values. First, they were obtained at strain-rates not lower than $10^{-7} \mathrm{~s}^{-1}$ and thus their application to geological processes may require extrapolations over many orders of magnitude in strain-rate. Second, the value of $A$ is not given in the Soviet paper and had to be estimated. This estimation was rendered difficult as the authors do not report the length of their specimens. Based on a comparison with other experiments done by the same group we have come to the conclusion that plausible range of specimen length results in the above value of $A$ being multiplied by a numerical factor between 0.8 and 1.3. Our choice is a compromise between these extremes.

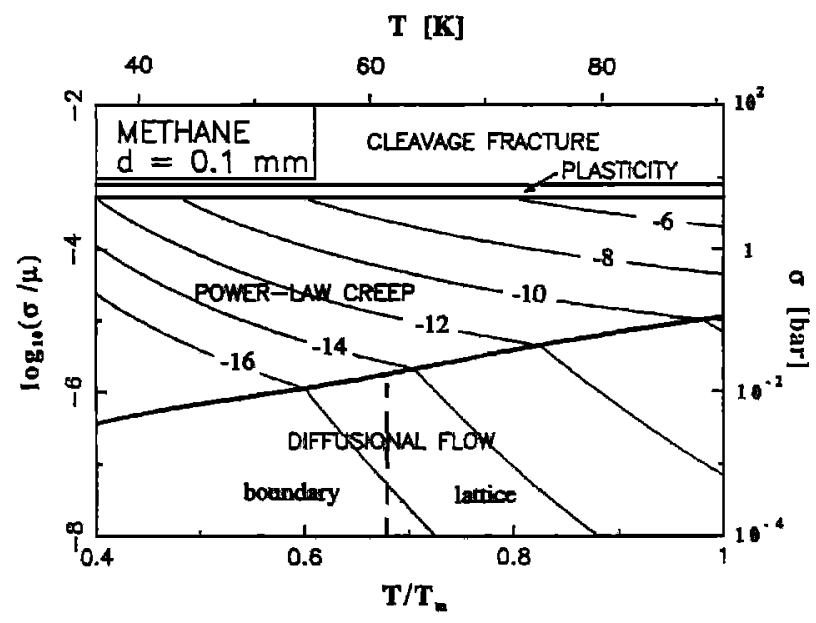

Fig. 1. Deformation map for solid methane for grain size $d=$ $0.1 \mathrm{~mm}$. The thin lines are contours of constant strain-rate labeled by the value of $\log _{10}(\xi)$, where $\varepsilon$ is the strain rate in $\mathrm{s}^{-1}$. This map may also be applicable to solid nitrogen in the homologous coordinates $\sigma / \mu$ and $T / T_{m}(\mu$ is the shear modulus and $T_{m}$ the melting teumerature).

A deformation map constructed from the above data is shown in Figure 1 for the mean grain size $d=0.1 \mathrm{~mm}$. Nothing is known about the latter in Triton's subsurface layers. Zent et al. [1989] argue in favor of meter-sized grains, but the validity of their argument has been brought into question by Kirk [1990]. The melting temperature of methane at ero pressure is $T_{m}=90.67 \mathrm{~K}$ and the shear modulus is $\mu=$
$20 \mathrm{kbar}$ [Bezuglyi et al. 1966]. For comparison, the stea modulus of water ice is $\mu=35$ kbar [Gammon et al. 1983) The lower value of $\mu$ for $\mathrm{CH}_{4}$ is consistent with weakex molecular bonding in solid methane. From Figure 1 it can be seen that power-law creep is indeed the dominant deformix mechanism, even for small grain sizes. Diffusional processm only become important at small grain sizes and stresses lower than 0.1 bar, which correspond to $\sim 20 \mathrm{~m}$ topograpby Triton. For this reason and because the main topographic relid on Triton is $\sim 1 \mathrm{~km}$, the uncertainty in grain size has no m-in impact on the conclusions of this paper with regand to the relaxation of topography. The fracture field is separated by only a narrow plasticity region from the creep field and in is therefore possible that cataclastic flow might be associnat with the highest topography on Triton. Litospheric streseas corresponding to $1 \mathrm{~km}$ topography are on the order of $4.6 \mathrm{bm}$.

As noted above, it is difficult to establish whether additional deformation processes operate in Triton's layers. Pressure solution creep requires the presence of tiy in the pores which given Triton's low temperatures seem unlikely for at least the upper few kilometers. Concerning dynamic recrystallization, nothing is known about the role, if any, of the formation of preferred texture in the flow of solv methane. Only future experimental efforts can shed light on this issue.

\section{Nitrogen}

The rheological data for nitrogen are much more scarce. Neither the fracture strength nor the yield stress have bee measured. However, both are unlikely to be very tifferea from methane's, given the similarity of the two maverials i terms of crystal structure and molecular bonding. This pcina is corroborated by the observation that the shear modalus of nitrogen has a value very close to the value of methane [Scett 1976]. The only direct rheological data are for volume diffusion [Esteve and Sullivan 1981]: $D_{\mathrm{ov}}=16 \times 10^{-8} \mathrm{~m}^{2} / 4$ $E_{\mathrm{y}}=8.6 \mathrm{~kJ} / \mathrm{mole}$. Given such meager informstion, the way of describing rheology of nitrogen is by using deformation map of methane in the homologous cocadianum Such procedure can be justified by the above mentioned similarity of the two ices and is rendered particularty appentive by the coincidence of shear moduli. Given that the sero pressure melting temperature $T_{m}=63.148 \mathrm{~K}$ of $\mathrm{N}_{2}$ is lowe than for $\mathrm{CH}_{4}$, nitrogen can be expected to be much softer methane at any particular stress and temperature.

\section{Consequences}

\section{Nitrogen and methane - mo solid-state interdiffusion}

The first question which can be addressed using the dut given above is the degree to which nitrogen and methane can be intermix od by solid-state diffusion. This is a potentily important issue as it relates to the problem of voluth redistribution in the last $\sim 3.5 \mathrm{Gyr}$, i.e., since the end of the intense tidal heating associated with circularization of Trum orbit [McKinnon 1984, Goldreich et al. 1989]. In large amounts of nitrogen well mixed with methene wail considerably soften rheology and aggravate the problen at preserving topography in nonwater ices. While is prospect at present to calculate the relative proportione of 
megen and methane on Triton, the degree to which they can diffuse can be estimated very simply. The upper limit on te coefficient of interdiffusion $D^{*}$ in an $n$-phase system is ive by the diffusivity of the fastest component. In the -aitrogen system the highest possible value is given by $D^{\prime}=D\left(N_{2}\right)$. In this case the mean square path $\sim\left(D^{*} t\right)^{1 / 2}$ over $t$ $=35 \mathrm{Gyr}$ is only $1 \mu \mathrm{m}$ at $37 \mathrm{~K}$ and $5 \mathrm{~cm}$ at $63.148 \mathrm{~K}$. enane and nitrogen, once separated, cannot be mixed in atid state by diffusion alone (a thermally driven separation have occured because $\mathrm{N}_{2}$ is preferentially volatilized with mect to $\mathrm{CH}_{4}$ ). This is consistent with the disequilibrium melage preferred by Lunine and Stevenson [1985]. On the wand, the generally short diffusion paths do not exclude te possibility of large grains advocated by Zent et al. [1989], at the relevant temperatures grain growth most likely cours through vapor transport.

\section{Detretim of topopraphy}

The discovery of a well preserved and diversified moguphy on Triton prompted the question whether such apography can survive over geological time in the two directly deervable components of Triton's surface, $\mathrm{CH}_{4}$ and $\mathrm{N}_{2}$. If this turns out to be impossible, then the natural implication will the layer of these ices is thin and topography is apported by water ice. From Figure 1 it is immediately clear the highest observed topography of $\sim 1 \mathrm{~km}$ cannot be apparted by methane ice alone (as mentioned above nitrogen tres even worse in this regard). Such topography coresponds to lithostatic stresses of -4.6 bar and in the $40-50$ I rage to strain-rates $\varepsilon \sim 10^{-12}-10^{-10} \mathrm{~s}^{-1}$. The RayleighTyylar relaxation time for a thin constant viscosity layer is [Pamentier and Head 1981]

$$
\tau=\left(3 / 4 \pi^{2} \dot{\varepsilon}\right)(\mathrm{h} / \mathrm{D})(\lambda / \mathrm{D})^{2}
$$

where $h$ is the height of the topography, $\lambda$ its horizontal wreleagth, and $D$ the thickness of the methane layer. In Fere 2 the wavelength of topography for which $t=3.5 \mathrm{Gyr}$ is plotted as a function of $D$. Only values $D$ greater than $h$ bud be considered since otherwise the estimate (1) ceases to be mingful. It is evident that only long-wavelength apography has any chance of surviving over several billion rears, e.g., for $T=40 \mathrm{~K}$ and $D=100 \mathrm{~m}, \lambda=100 \mathrm{~h}$, whereas tor $\mathrm{D}=10 \mathrm{~km}, \lambda=10^{5} \mathrm{~h}$ is required to achieve this. For $\mathrm{T}=$ $50 \mathrm{~K}$ (which may corresponds to regions of lower albedo), heady for $D=100 \mathrm{~m}$ only topography with $\lambda$ greater than $1000 \mathrm{~h}$ can survive. For $\mathrm{T}=60 \mathrm{~K}$, no kilometer-scale apopraphy can survive over 3.5 Gyr. Such estimates, despite teir limimions, allow several conclusions:

1. Short-wavelength topography such as cliffs and ridges the fomed recently or is supported by water ice.

2. The largest observed impact crater $(\lambda=27 \mathrm{~km})$ could be preserved in methane only if the methane layer is igmificantly thinner than $1 \mathrm{~km}$ and the subsurface maperantes are low $(T-40 \mathrm{~K})$. In fact, this is unrealistic, wach a large crater would excavate below the thin layer. Also, if the viscosity decreases with depth, the wavelength components will relax away faster than predictod by Eq. (1).

Thus it seems almost certain that topography is supported 7 aner-based ice (e.g., ondinary water ice, ammonia or clathrate).

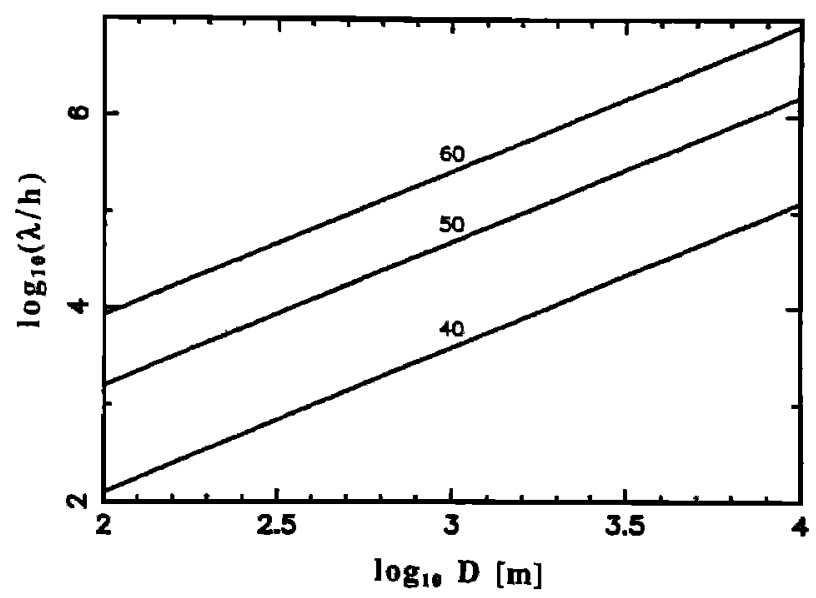

Fig. 2. The ratio of the wavelength of topography to topographic height $\lambda / \mathrm{h}$ for topography which can be supported over $3.5 \mathrm{Gyr}$ by a layer of solid methane as a function of layer thickness $D$. Only values $h$ much smaller than $D$ should be considered. The curves are labeled by the values of temperature at the base of the methane layer. Owing to $n=3$ in the creep law, the curves are independent of $h$ in these coordinates.

\section{The opposition effect (or lack thereof?)}

The opposition effect is defined as an enhancement in the logarithmic brightness of an object when observed at phase angles $\leq 7^{\circ}$, in excess of that predicted by a linear extrapolation of the brightness vs phase relation from larger phase angles. Groundbased observations combined with Voyager data suggest that the opposition effect on Triton is very weak or absent [Smith et al. 1989]. As this effect is usually associated with the porous structure of the optically active layer, a possible explanation would involve pressureless sintering of the uppermost layers. This process would be most efficient in the nitrogen frost. The rate at which this surfacetension driven sintering proceeds is given by the formula [Ashby 1988]

$$
\phi=72 \mathrm{C}\left(1-\phi_{0}\right) \mathrm{D}_{v} \gamma \Omega / \mathrm{kTd}^{3}
$$

where $\phi_{0}$ and $\phi$ are the starting and actual porosity, respectively, $C=\phi_{0} /\left(\varphi_{0}-\phi\right)$, and $\gamma$ is the surface energy. Given the similarity of elastic moduli of nitrogen and water ice, it seems plausible to adopt for nitrogen $\gamma=0.1 \mathrm{~J} \mathrm{~m}^{-2}$, the value for water ice. Assuming $\phi_{0}=0.36, \phi=0.3$ and using diffusion data for nitrogen, one obtains the timescale for compaction at $\mathrm{T}=37 \mathrm{~K}, \tau_{\mathrm{c}}[\mathrm{s}]=-\phi / \dot{\phi}=5 \times 10^{23}(\mathrm{~d} / 1 \mathrm{~m})^{3}$. For annealing to proceed on a timescale of Triton's seasons ( $\tau_{c}$ $\sim 10^{2} \mathrm{yr}$ ), $\mathrm{d}$ smaller than $20 \mu \mathrm{m}$ is required. While this is a plausible range of grain sizes for freshly deposited frost, the possibility of a rapid grain growth [Zent et al. 1989] presents a complicating factor (it is worth noting that grain growth by itself does not remove porosity and thus should not contribute to the decrease in the opposition surge). We conclude that sintering offers a speculative explanation at best for the weakness of the opposition effect. Recent reevaluation of the groundbased observations suggests that the opposition effect, while weaker than for other icy satellites, is present on Triton after all (A. Harris, personal communication). New 
observations would be of paramount help in clarifying the picture.

\section{Summary}

Based on the existing theological data it is concluded that on Triton solid methane and most likely nitrogen are too soft to support any topography over billions of years. Topographic support by water ice-rich materials seems almost certain. Nitrogen and methane do not intermix by solid-state diffusion. The weakness (absence?) of the opposition effect on Triton can be explained by annealing of nitrogen grains, provided the latter have sizes characteristic of freshly deposited frost. Recommendations for further research include: 1. Creep measurements of solid nitrogen; 2. Better understanding of grain growth in solid nitrogen; 3 . Clarification of the opposition behavior.

Acknowledgements. Thanks are due to Bill McKinnon and two anonymous referees for their careful reading of the manuscript and helpful comments. This work has been supported by the NASA Planetary Geophysics Grant NAGW185. Contribution number 4881 from the Division of Geological and Planetary Sciences, California Institute of Technology, Pasadena, California 91125.

\section{References}

Ashby, M. F., Background reading: Hot isostatic oressing and sintering, Engineering Deparment, Cambridge, 1988.

Ashby, M. F. and R. A. Verall, Micromechanisms of flow and fracture, and their relevance to the rheology of the upper mantle, Phil. Trans. R. Soc. Lond. A, 288, 59$95,1978$.

Bezuglyi, P. A., N. G. Burma, and R. Kh. Minyafaev, Elastic constants of polycrystalline methane in the temperature range 14.4-77 K, Sov. Phys. Solid State, 8 . $596-600,1966$.

Bolshutkin, D. N., L. I. Barisova, and A. V.Leonteva, Creep of crystalline methane, Sov. Phvs, Solid State 10, 12481249, 1968.

Broadfoot, A. L. and 21 collaborating authors, Ultraviolet Spectrometer observations of Neptune and Triton, Science, 246, 1459-1466, 1989.
Chezeau, J. M. and J. H. Strange, Diffusion in molecalu crystals, Phys, Rep. 1, 1-92, 1979.

Esteve, D. and N. S. Sullivan, NMR study of self-diffacion i solid $\mathrm{N}_{2}$, Solid State Commun., $39,969-971,1981$.

Gammon, P. H., H. Kiefte, and M. J. Clouter, Blasto constants of ice samples by Brillouin spectroscopy, 1 Phys. Chem., 87, 4025-4029, 1983.

Goldreich, P., N. Murray, P. Longaretti, and D. Benfild Neptune's story, Science, 245, 500-504, 1989.

Haynes, W. H., Shear strengths of solid methane and argen 8 kbar at $77 \mathrm{~K}$, L. Phys. Chem. Solids 32 791-7m. 1971.

Kirk, R. L, Diffusion kinetics of solid methane and nitro Implications for Triton (abstract), Lunar and Pinsta Science Conference, 21, 631-632, 1990.

Leonteva, A. V., Yu. S. Stroilov, E. E. Lakin, and D. K Bolshutkin, Zero-point oscillation energy effect on deformation in solidified gases, Phys, Stat. Sol. 42 513. $549,1970$.

Lunine, J. L. and D. J. Stevenson, Physical state of volutha on the surface of Triton, Narure, 317, 238-240, 1985.

McKinnon, W. B., On the origin of Triton and Phuta, Nman 311, 355-358, 1984.

Parmentier, E. M. and J. W. Head, Viscous relaratice impact craters on icy planetary surfaces: Determination viscosity variation with depth, Icarus $47,100-111,1981$.

Scott, T. A., Solid and liquid nitrogen, Phys. Rep. 3 , $157,1976$.

Smith, B. A. and 64 collaborating authors, Voyager 2 * Neptune: Imaging science results, Science, 246,100 1449, 1989.

Zent, A. P., C. P. McKay, J. B. Pollack, and D. P. Cruikshank, Grain metamorphism in polar nitrogea ice Triton, Geophys. Res. Lett. 16, 965-968, 1989.

Janusz Eluszkiewicz and David J. Stevenson, Divisioa of Geological and Planetary Sciences, California Institutio Technology, Pasadena, CA 91125, USA.

(Received March 29, 1990; revised June 18, 1990; accepted June 20, 1990.) 